\title{
Face and content validity and acceptability of the Swedish ICECAP-O capability measure: Cognitive interviews with 70 -year-old persons
}

\author{
Susanne Gustafsson, ${ }^{1,2}$ Helena Hörder, ${ }^{2,3}$ Isabelle Ottenvall Hammar, 1,2 Ingmar Skoog ${ }^{3}$ \\ ${ }^{1}$ Department of Health and Rehabilitation, Institute of Neuroscience and Physiology, Sahlgrenska \\ Academy; ${ }^{2}$ Centre for Ageing and Health (AgeCap); ${ }^{3}$ Department of Neuropsychiatric \\ Epidemiology, Institute of Neuroscience and Physiology, Sahlgrenska Academy, \\ University of Gothenburg, Sweden
}

\begin{abstract}
This study is part of a project that aims to culturally adapt the Investigating Choice Experiments for the Preferences of Older People-CAPability Index (ICECAP-O) for use in research and health and social care in Sweden. The objective was to evaluate face and content validity and acceptability. Eighteen 70-year-old
\end{abstract}

Correspondence: Susanne Gustafsson, Department of Health and Rehabilitation, Institute of Neuroscience and Physiology, Sahlgrenska Academy, University of Gothenburg, Box 455, SE 40530 Göteborg, Sweden.

Tel.: +46.733.92.50.97

E-mail: susanne.gustafsson@neuro.gu.se

Acknowledgements: the authors want to thank Therese Rydberg for help with the recruitment of participants. Their warmest thanks to the participants from the $\mathrm{H} 70$ study for contributing to the cultural adaptation of the ICECAP-O measure and further understanding of the concept of capability.

Key words: Quality of life; Older adults; Elderly; Geriatric assessment; Self-assessment.

Contributions: SG and $\mathrm{HH}$ jointly initiated the study and planned the research design. SG collected the data and was the primary author of the manuscript. SG, $\mathrm{HH}$, and $\mathrm{IOH}$ were all engaged in data analysis. IS is the research director of the $\mathrm{H} 70$ study, guided the research process and provided valuable support.

Conflict of interest: the authors declare no potential conflict of interest.

Funding: the authors thank the Swedish Research Council (201502830), the Swedish Research Council for Health, Working Life and Welfare (AGECAP 2013-2300, 2013-2496, 2013-1202), and Sahlgrenska University Hospital (ALF) for financing and supporting the study.

Received for publication: 19 December 2016.

Revision received: 4 October 2017

Accepted for publication: 13 November 2017.

This work is licensed under a Creative Commons AttributionNonCommercial 4.0 International License (CC BY-NC 4.0).

(C) Copyright S. Gustafsson et al., 2018

Licensee PAGEPress, Italy

Health Psychology Research 2018; 6:6496

doi:10.4081/hpr.2018.6496 community-dwelling persons participated in cognitive interviews. A standardized classification scheme was used to quantify any identified response problems, and a thematic analysis was applied to capture participants' perceptions of included attributes and experiences of completing the measure. The results show that three participants $(18 \%)$ had problems completing ICECAP-O, and that judged problems occurred for five $(6 \%)$ of participants responses in the standardized classification scheme. Most participants perceived the attributes as understandable even though the meaning of Control brought some uncertainty. ICECAP-O seems to measure what it is supposed to measure, quality of life (QoL) with a capability approach, and acceptability is satisfactory. ICECAP-O has potential for becoming a valuable addition to the supply of QoL measures in research and health and social care in Sweden. However, we recommend further research on more diverse groups of older persons.

\section{Introduction}

The Investigating Choice Experiments for the Preferences of Older People-CAPability Index (ICECAP-O) (Grewal et al., 2006) is a measure of quality of life (QoL) with a capability approach tailored for older persons. It aims to capture an individual's ability to do and be the things that they regard as important in life. In doing so, it focuses on general attributes of QoL rather than health states. Such a measure is especially valuable for programs targeting public health, interventions crossing health and social care boundaries, multidisciplinary actions, and economical evaluation (Coast, Flynn, et al., 2008). However, such measures in the context of research and health and social care in Sweden are lacking and therefore should be addressed. Hence, this study is part of a project to culturally adapt the ICECAP-O capability index for Sweden.

Thousands of measures of QoL exist, both generic and healthrelated (Makai, Brouwer, Koopmanschap, Stolk, \& Nieboer, 2014). Most include health status and predetermined functions, but fail to include possibilities to actually be or do things that are valued by the individual. A broader approach to QoL appears particularly relevant at old age when health may not be considered the most important factor (Garratt, Schmidt, Mackintosh, \& Fitzpatrick, 2002). Capability is considered as a more appropriate evaluative space than function (health status) because two people may both have the specific function, but only one may have the actual possibility to fulfil this function in action or in being (i.e., capability).

Capability could be considered as the effective possibilities a 
person has to convert one's resources to achieve a desired goal, such as health (Sen, 1993). Health is a dynamic state that could be influenced by internal factors such as a person's values, beliefs and functions, and external factors shaping the circumstances for converting ones' resources into health (Prah Ruger, 2010). Thus, capability refers to what individuals are actually able to be or do in their environment (Sen, 1993). Older persons might not have equal opportunities to enjoy good health due to factors at an individual (micro), group (meso), or societal (macro) level. The combination of individual factors including physical health and cognition, group factors such as family and community, and societal factors exemplified by laws and healthcare services, may at any given time form the basis for an individual's capability set, i.e, what an individual actually can or cannot do (Sen, 2009). For instance, two older persons may value to be as independent as possible. One of the older person's limbs works well resulting in few needs of resources to be mobile, while the other person may have an impairment/disability resulting in an increased need of resources to achieve the same level of mobility.

The ICECAP-O was developed according to in-depth interviews with older persons to capture QoL beyond health status (Grewal et al., 2006). In its final English version, it contains five attributes found to be important for general QoL; Attachment love and friendship; Security - thinking about the future without concern; Role - doing things that makes you feel valued; Enjoyment - enjoyment and pleasure; and Control - independence (Coast, Flynn, et al., 2008). Psychometric evaluation work of the ICECAP-O is ongoing (Coast, Peters, Natarajan, Sproston, \& Flynn, 2008; Horwood, Sutton, \& Coast, 2014), one recent publication covers reliability in the Swedish context (Hörder, Gustafsson, Rydberg, Skoog, \& Waern, 2016).

Implementing a questionnaire developed and tested in a specific context (e.g. the UK) in a dissimilar context (e.g. Sweden), requires a cross-cultural adaptation. Cross-cultural adaption strives to produce equivalency based on content. If the process is of sufficient quality, then other psychometric properties may be retained. Three criteria in a QoL measure in need of attention in a cross-cultural adaptation are face and content validity and acceptability. Face validity refers to whether an instrument appears to be measuring the domain of interest, and content validity is a judgement about whether instrument content adequately covers the domain of interest (Fitzpatrick, Davey, Buxton, \& Jones, 1998). Acceptability is defined as the extent to which an instrument is acceptable to patients and includes, for example, format and administration time. This study is part of a project that aims to culturally adapt the ICECAP-O for use in research and health and social care in Sweden. To date, two studies nested within a larger study called H70 (Rinder, Roupe, Steen, \& Svanborg, 1975) have been initiated. The current study aims to evaluate face and content validity and acceptability of the ICECAP-O capability index for use in Sweden.

\section{Materials and Methods}

\section{Design}

To meet the study objective, a cognitive interviewing technique was used (García, 2011). Cognitive interviewing, a generic concept comprising a group of techniques based on theories of cognitive psychology, is well established in health research. The technique aims to enhance the quality of research questionnaires by providing a means to assess respondents' understanding. In this study, we used the concurrent think aloud technique, in which participants' thought processes were verbalized during their attempts to complete the translated ICECAP-O measure (García, 2011). Furthermore, in a debriefing session after the ICECAP-O questionnaire was completed, we applied retrospective probes to facilitate narratives on participant's thoughts of completing the questionnaire and its content (Willis \& Miller, 2011). The Regional Ethical Review Board in Gothenburg approved this study (reference \#T139-15) and written informed consent was obtained from the participants.

\section{Study population}

Thirty 70-year-old community-dwelling persons were asked about partaking in this study, out of which 18 agreed to participate. They had all, prior to this study, participated in a larger population study named H70 (Rinder et al., 1975) during the first two months of 2015. H70 is a comprehensive population-based study of health and related factors among older persons. Generally, the participants were in good health even though minor disabilities such as impaired vision, body aches, and the use of a single walking aid occurred. They all lived in the same medium-sized Swedish city covering both the city center as well as more rural areas and lived in either apartments or self-owned houses. One participant failed to attend the scheduled interview resulting in 17 interviews (10 women). Table 1 shows participants' living conditions and education levels.

\section{Instrument}

The ICECAP-O is a self-administered questionnaire measuring QoL in its broadest sense with a capability approach (Grewal et al., 2006). The questionnaire contains five attributes: Attachment, Security, Role, Enjoyment, and Control, each with four-level response options that are described as statements representing: none, a little, a lot, and full capability. Also, a total index score, based on a tariff computed from population-based values in

Table 1. Overview of the 70-year-old study participants $(n=17)$.

\begin{tabular}{lccc}
$\begin{array}{l}\text { Participant } \\
\text { (number) }\end{array}$ & Sex & $\begin{array}{c}\text { Living } \\
\text { alone }\end{array}$ & $\begin{array}{c}\text { Higher } \\
\text { Education }\end{array}$ \\
1 & F & No & Yes \\
2 & M & No & No \\
\hline 3 & F & No & Yes \\
4 & M & No & Yes \\
\hline 5 & F & No & No \\
6 & F & No & No \\
\hline $7 *$ & F & Yes & No \\
8 & M & No & Yes \\
\hline 9 & M & No & Yes \\
10 & M & No & No \\
\hline 11 & F & No & No \\
12 & F & Yes & Yes \\
\hline 13 & F & No & No \\
14 & M & No & Yes \\
\hline 15 & F & No & Yes \\
16 & M & No & Yes \\
\hline 17 & F & No & No \\
\hline
\end{tabular}

*Interview excluded owing to technical error; "Initiated or completed college/university. 
the UK is available. Values are anchored with a best-worst scaling, ranging from 0.00 (no capability) to 1.00 (full capability) (Coast, Flynn, et al., 2008). Both the English and Swedish versions of the ICECAP-O capability measure are freely available online (http://www.birmingham.ac.uk/research/activity/mds/projects/Ha PS/HE/ICECAP/ICECAP-O/index.aspx).

\section{Procedure}

Study data were collected from April to June 2015. It was preceded by a translation of the original English ICECAP-O version into Swedish, which involved independent forward and backward translations by two independent authorized translators (Guillemin, Bombardier, \& Beaton, 1993). Any discrepancies in the translation were resolved through discussion with the authors. Thereafter, a panel of experts consisting of persons with knowledge in gerontology, geriatrics, psychiatry, and occupational and social sciences compared the Swedish and the English versions and agreed upon a final version.

Next, an independent research assistant strategically selected 30 persons who had participated in the H70 study from January and February 2015. The aim was to gather a heterogeneous sample consisting of an equal amount of men and women with varying demographics who had not previously been asked to participate in any additional study within the H70 study. Letters were sent asking the selected persons to participate in this study. Invited persons also received a description of the study, how it was to be conducted, and what was expected of those consenting to participate. The letter stressed the fact that participation was voluntary. This was followed by a telephone call approximately 1-2 weeks later. During the call, the persons were informed verbally about the study and they were given the opportunity to ask further questions. Eighteen of the 30 invited persons $(60 \%)$ agreed to participate; one person failed to attend the interview, resulting in a total of 17 participants. Reasons for nonparticipation included no public phone number registered $(n=2)$, unreachable by phone $(n=4)$, not interested $(n=3)$, and have no time $(n=3)$. The number of participants $(\mathrm{n}=17)$ was judged sufficient because data saturation was reached. No new information emerged during the last two interviews, which was why no additional participants were recruited. The interviews took place in a private meeting room at the University of Gothenburg or, if preferred, at the participant's own home. Two participants choose the latter alternative. The translated version of the ICECAP-O was administered after the participants had provided written informed consent. The participants were instructed to read aloud each question and verbalize their thoughts while answering the questions. In line with a similar study in the UK (Horwood et al., 2014), participants were told not to plan ahead what they were going to say, but to act as if they were alone in the room thinking aloud to themselves. The first author (SG), who conducted all interviews, then sat out of the participant's line of view. The participants were not disturbed or interrupted after they had begun completing the measure, unless they paused for a period longer than 10-15 seconds, in which case the researcher reminded the participant to keep thinking aloud. All other interactions between the two parties during the completion of the questionnaire were minimized. After the participant had completed the questionnaire, the interviewer moved to a chair facing the participant for a debriefing session. Here, a conversation aiming at capturing the participants' thoughts on completing the ICECAP-O was initiated by meaning-oriented and evaluative retrospective probes such as, What did you think about the first question?; How did you find the answering alternatives?; What does independence mean to you?

\section{Analysis}

All 17 interviews were audio recorded, but a technical error occurred in one interview resulting in 16 interviews being transcribed verbatim. The transcribed interviews were analyzed (a) using a standardized classification scheme to identify and quantify response problems (the think-aloud part of the interview), and (b) qualitatively thematically analyzed to explore the kind of the problems that the participants experienced when completing the measure (both the think-aloud part and the debriefing session).

The standardized classification scheme used to quantify any identified response problems during the think-aloud part of the interview was identical to the one used in a similar study of content validity of the ICECAP-O in the English context (Horwood et al., 2014). Each of the five ICECAP-O attributes, in all interviews, were examined by three of the authors ( $\mathrm{SG}, \mathrm{HH}$, and $\mathrm{IOH}$ ) for possible problems according to four areas of response problems; comprehension (i.e., any misunderstanding of a word, phrase or response option); retrieval (i.e., a recall problem from memory of information used to prepare an answer to the question); judgment (i.e., recalled experiences are irrelevant or inadequate); and response (i.e., participant's response is inconsistent with the personal experience expressed or the desired response is missing from the response choices). These four areas of response problems were, in turn, based on the question and answer model developed by cognitive psychologists (Willis \& Miller, 2011). Additionally, we noted if participants showed signs of struggling with an attribute (e.g., re-reading the question several times, or questioning how sensible the question was), but were finally able to answer the question. A response problem was considered to be present if two or three of the raters/authors independently identified a problem. Finally, to assess inter-rater agreement, percent-agreement (PA) (McHugh, 2012; Svensson, 2001) was calculated for (i) the five ICECAP-O attributes, and (ii) all sections in the classification scheme (overall PA) according to the following formula:

\section{$n$ totally agree \\ (i) $n$ segments within each of the five ICECAP-O attributes or (ii) all sections in the classification scheme}

The thematic analysis conducted on data from the whole interview was, conversely, qualitative in its nature and based on the method developed by Braun and Clark (Braun \& Clarke, 2006). The transcribed interviews were read several times by all authors independently to look for patterns and to interpret explicit meaning. In line with the study of Horwood et al. (2014), extracted data were first categorized into two broad groups of data. One group comprised data dealing with information on the concept of capability; the other covered specific information regarding the ICECAP$\mathrm{O}$ measure, e.g., the wording of an attribute or the assessment situation. The first author (SG) coded and interpreted the data. The other authors contributed suggestions for alternative interpretations and presentation of results. Any discrepancies between the authors was discussed and solved in consensus.

\section{Results}

The average ICECAP-O index value for all participants was 0.8148 . Values ranged from 0.5313 to 1 . One participant estimated their capability at the highest level for each attribute. There was no significant difference in the index values according to demographics (results not shown). 
Participants spent an average of 7.29 minutes to complete the ICECAP-O measure while thinking-aloud (range $4.21-11.30$ minutes). The whole cognitive interview, both the think-aloud part and the debriefing session, averaged 18.53 minutes (range $9.42-$ 35.27 minutes).

\section{Description of problems identified by the raters}

The quantitative scoring of the transcribed think-aloud part of the interviews identified between zero and three problematic responses per participant. As is shown in Table 2, three participants $(18 \%)$ were identified as experiencing problems in completing one or more of the five items in the ICECAP-O measure. Three additional participants struggled, but were judged to answer the attribute appropriately, resulting in six out of 17 participants (35 $\%$ ) having some difficulty completing the measure.

In the standardized classification scheme, judged problems occurred for five $(6 \%)$ of participants responses. Problems with comprehension and judgment were detected twice each, while a response problem occurred once and no problems were identified concerning retrieval. In addition, problems where participants struggled were identified eight times, half of which occurred for Control $(n=4)$. Table 3 shows the distribution of the judged problems according to the ICECAP-O attributes. It is evident that Control is the attribute vitiated with the majority of judged problems $(n=4)$. Enjoyment had one judged problem, while Role, Attachment, and Security had none. Nevertheless, Role and Enjoyment each had two problems where participants struggled. The overall PA for the three raters of all participants' responses in the standardized classification scheme was $93 \%$. The PA for each of the five ICECAP-O areas was as follows; Attachment $99 \%$, Security $98 \%$, Role $98 \%$, Enjoyment $94 \%$, and Control $78 \%$, demonstrating considerable agreement but least for Control.

\section{Participants' experiences of completing the ICECAP-O measure}

Most participants perceived the items as understandable and experienced little or no problem in answering them. Even so, some expressed that they found the items somewhat diffuse or broad, consisting of a mix of aspects. The depth of the items, and the emotions it aroused, were experienced as surprising. One participant said, these questions feel both unfamiliar and private for me to answer. Others argued that the items contained strong expressions requiring reflection, and recommend not answering them too quickly. Participants claimed that one's personality played an important role in how items were answered, e.g., cheerful or downhearted personalities will be mirrored in the answers, hence identifying character as a factor affecting self-perceived capability. In addition, expressions of gratitude were conveyed that someone was interested in and cared about listening to participants' reasoning about these life issues. The last ICECAP-O attribute Control, operationalized as able to be completely independent, was the item most frequently discussed during the debriefing sessions. Uncertainty about the meaning of independence was articulated as well as a request for clarification. Some participants found it difficult to determine if they could be fully independent because they had lived together with their spouse for decades, and others stated that no one could be totally independent. This quote is an example, Independence was the hardest to answer. You are dependent on other people. No one can be completely independent. One participant further reported that they refrained from answering the item about independence because its implication was unclear. Finally, a highlighted view concerning the design of the ICECAP-O measure was the text size; it should be enlarged, especially for visually disabled persons. Other single suggestions for improvements were more response options and items covering health and physical activities.

Table 2. Frequency and type of response problem per study participants.

\begin{tabular}{|c|c|c|c|c|c|c|c|}
\hline No. & $\begin{array}{c}\text { No substantive } \\
\text { problem } \\
\text { identified* }\end{array}$ & Comprehension & $\begin{array}{l}\text { Identified } \\
\text { Retrieval }\end{array}$ & $\begin{array}{l}\text { oblems } \\
\text { Judgement }\end{array}$ & Response & $\begin{array}{c}\text { Total substantive } \\
\text { problem }\end{array}$ & $\begin{array}{l}\text { Struggled but } \\
\text { answered } \\
\text { correctly }\end{array}$ \\
\hline 1 & 5 & - & - & - & - & - & - \\
\hline 2 & 2 & 1 & - & 1 & 1 & 3 & 1 \\
\hline 3 & 5 & - & - & - & - & - & - \\
\hline 4 & 5 & - & - & - & - & - & 1 \\
\hline 5 & 4 & - & - & 1 & - & 1 & 3 \\
\hline 6 & 5 & - & - & - & - & - & - \\
\hline 8 & 5 & - & - & - & - & - & - \\
\hline 9 & 4 & 1 & - & - & - & 1 & 1 \\
\hline 10 & 5 & - & - & - & - & - & - \\
\hline 11 & 5 & - & - & - & - & - & - \\
\hline 12 & 5 & - & - & - & - & - & 1 \\
\hline 13 & 5 & - & - & - & - & - & - \\
\hline 14 & 5 & - & - & - & - & - & - \\
\hline 15 & 5 & - & - & - & - & - & 1 \\
\hline 16 & 5 & - & - & - & - & - & - \\
\hline 17 & 5 & - & - & - & - & - & - \\
\hline Total & 75 & 2 & 0 & 2 & 1 & 5 & 8 \\
\hline
\end{tabular}

*Number of ICECAP-0 attributes not vitiated with a response problem; \#Total number of ICECAP-0 attributes vitiated with a response problem. 


\section{Participants' perceptions of the ICECAP-O attributes}

Regarding Attachment, participants stated that their partners, families and relatives, and friends were important components. An extended relationship with a partner was seen as an important source of love and friendship even though some meant that a longterm relationship could lead to routine and an uneven distribution of desires for closeness, influencing capability. Having good relations and frequent gatherings with the immediate family and relatives rendered high estimates for Attachment, while a lower estimate was described for the opposite, often explained by relatives living far away. This quote is an example of the latter, My grandchildren [pause] they live so far away. I hear about others who pick them up at day-care and spend afternoons with them and I wish I could do that too. The importance of friends for QoL was highlighted, as well as the significance of both close and more distant friends. Concerns were raised regarding the problem of the tendency for social networks to shrink with ageing, and the difficulty in making new friends after retirement, both issues negatively affecting capability. Participants also expressed the view that the attribute implied the individual's responsibility for interchange. In order to receive the love and friendship you need and want, you also have to give.

When discussing Security and thoughts about the future, participants reasoning varied along a broad continuum from, I am not worried at all; it [future] is not that lengthy, to great concerns for many aspects of life, Well, I worry a lot [pause] for mine and my husband's health, our children, and for the development in society. As the latter quote denotes, thoughts about the future were often linked to one's own and relatives' health, thus, this was a feature identified as affecting capability. The primary concern for own health was dementia, to lose one's memory and be unable to communicate, and to therefore become dependent. However, other issues were also raised: the economy with decreased pensions, being unable to ascend and descend home stairs or tend to one's garden, and shortage of adequate accommodation for older persons if relocation is necessary, factors impacting capability. In addition, thoughts often reflected on stressful societal situations: environment and pollution, violence, poverty, and segregation.

The attribute Role consists of an estimate if you are able to do things that make you feel valued. Participants primarily described this as receiving appreciation from others in shared activities, i.e., being seen and therefore self-generating own positive emotions.
They said this occurred when doing things with and for their family and friends, exemplified by the quote, I feel valuable when he [grandchild] says 'grandfather' when we play; it's enough to feel good for a whole week. In addition, participants meant that one can feel valuable in solitary activities such as reading, as well as in engagement in associations and voluntary work. The need for appointments in one's agenda in order to feel valued was advocated, as well as a view that feeling valued was easier when still employed. Descriptions of being forced to downgrade, to be content with fewer feelings of value were expressed. This situation induces reduced capability and was exemplified by increasing age with decreased strength and mobility, and other ailments or disease.

Enjoyment was described in terms of being active, e.g., eating good food, reading, listening to music, vacationing, and spending time in nature. Again, joint events with spouse, family, and friends were described as sources of enjoyment, as were opportunities to give others joy and pleasure through acts without self-interest, such as conducting music sessions for people with disabilities at a group home. A relationship with one's partner built on tenderness and affection, and possibility for sex was also mentioned as significant. Moreover, descriptions of factors limiting capability emerged, including one's own or a significant other's illness or disease, age, and decreased endurance, as well as poor economy. One participant said, One must of course think of one's age, what you can cope with doing, and how you feel.

Finally, for the attribute Control, persons rated their QoL, indicating if they can be completely independent. Independence was found to encompass aspects of emotional and financial independence, as well as not needing support for personal care and daily activities. One view expressed was that we all live in a context dependent on the climate, laws and regulations, and the range of available accommodations in which no one can be fully independent, affecting each and everyone's capability. Additionally, everyone is dependent on others for communion, confirmation, and the exchange of services, exemplified by this quote, I always depend on my friends and my husband to cope with the house. Interpretations of independence included being able to be who you want to be and make autonomous decisions. It also comprised to be able to do the things you want to do when you want to do them and again, the main hindrance to capability was reported to be own health status.

Table 3. Frequency and type of response problem per attribute/capability concept.

\begin{tabular}{|c|c|c|c|c|c|c|c|}
\hline $\begin{array}{l}\text { No. } \\
\text { capability } \\
\text { concept }\end{array}$ & $\begin{array}{c}\text { No substantive } \\
\text { problem } \\
\text { identified* }\end{array}$ & Comprehension & Identified & Judgement & & $\begin{array}{c}\text { Total substantive } \\
\text { problem }\end{array}$ & $\begin{array}{l}\text { Struggled but } \\
\text { answered } \\
\text { correctly }\end{array}$ \\
\hline
\end{tabular}

Attachement

16

$n=16$

\begin{tabular}{|c|c|c|c|c|c|c|c|}
\hline $\begin{array}{l}\text { Security } \\
n=16\end{array}$ & 16 & - & - & - & - & - & - \\
\hline $\begin{array}{l}\text { Role } \\
n=16\end{array}$ & 16 & - & - & - & - & - & 2 \\
\hline $\begin{array}{l}\text { Enjoyment } \\
n=16\end{array}$ & 15 & - & - & 1 & - & 1 & 2 \\
\hline Total & 75 & 2 & - & 2 & 1 & 5 & 8 \\
\hline
\end{tabular}

*Number of attributes not vitiated with a response problem; \#Total number of identified response problems. 


\section{Discussion}

Overall, the attributes in the ICECAP-O measure were interpreted as anticipated and the extent to which one struggled to complete the measure was moderate. The results showed that three participants $(18 \%)$ had problems completing the measure compared with $25 \%$ reported for the original English version (Horwood et al., 2014). Regarding participants responses in the standardized classification scheme, judged problems occurred for $6 \%$, where the corresponding figure for the original version was $7 \%$ (Horwood et al., 2014). The comparable results for the Swedish and the original English version of the ICECAP-O were affirmative, suggesting that the measure works equivalent in both contexts and is suitable for continued development and implementation in the Swedish context.

Most participants perceived the attributes as understandable even though Control - (i.e., independence) - caused some uncertainty and desire for clarification. This results contrasts with that of Coast et al. (2008), who found Security to be the most disputed attribute among participants in their study, and van Leeuwen et al. (Van Leeuwen et al., 2015), where Role was found to be the most difficult to understand. In addition, Control was the attribute with the highest number of judged problems and the lowest PA value for the three raters $(78 \%)$. One possible explanation is that participants in our study were community-dwelling 70-year-old persons who remained in relatively good health with no or little experience of being dependent in activities of daily life, characterizing the third age (Baltes \& Smith, 2003). Hence, they did not have an obvious situation of dependence to relate to when completing the measure, which may have hampered full comprehension of the attribute. Furthermore, participants claimed that Control implied ability to make autonomous decisions, i.e., to exercise self-determination. This purport is consistent with the description of Control in the original work (Grewal et al., 2006), but this implication does not emerge in the attributes text in ICECAP-O. Even if it has previously been shown that independence is experienced as one of the core categories in self-determination within the context of community-dwelling older persons (Ekelund, Dahlin-Ivanoff, \& Eklund, 2014), the overlap of the concepts may have contributed to the challenge of grasping Control, a topic also raised by others (Coast, Flynn, et al., 2008). Moreover, financial and emotional independence were put forward as additional meanings of this attribute, indicating ambiguity, which may have contributed to wishes for clarification. An issue like this, that arises in one language but not in another, must be reconciled, and judgements made concerning whether it is due to errors of translation, cultural disparities, or artefacts of different approaches to the conduct or analysis of the cognitive interviews (Willis \& Miller, 2011). We find our result for Control to be, at least partly, due to the selection of participants. However, it raises concerns about cross-cultural equivalence and indicates a need to clarify Control for the use of ICECAP-O in the Swedish context.

One's own and significant others' health status, social network, and economy emerged as important factors affecting participants' capabilities. This is consistent with the original work by Grewal et al. (2006), who described poor health, poor economy, and bereavement as negative influences on several of the five attributes, and an Australian study (Couzner, Ratcliffe, Lester, Flynn, \& Crotty, 2013), that found that those with higher income have higher ICECAP-O scores on average than those with lower income. Another factor negatively impacting capability described in the original work (Grewal et al., 2006), but not raised in our study, is the pro- vision of informal care. This distinction can be explained by the age of the participants in this study, the absence of the oldest old, where situations of informal care are more common. This reasoning is supported by findings by van Leeuwen et al. (Van Leeuwen et al., 2015), where the issue of providing informal care was raised by their participants, who were aged $\geq 75$-years. In addition to the original work (Grewal et al., 2006), participants in this study also considered negative factors in society as a whole impacting capability: violence, poverty, and segregation. These factors were primarily thought to impact Security, and may be a sign of increased awareness regarding macro factors impacting a person's capability consistent with the discourse on justice raised by Sen et.al. (Hammell, 2015; Sen, 2009).

All attributes in the ICECAP-O measure were discussed in terms of both significance/meaning and disincentives for capability, making them relevant and essential for the purpose of the measure. One suggestion raised by participants was to incorporate additional items covering health and physical activity. However, simultaneously, they discussed different aspects of their own health status and found it to affect present attributes. By doing so, aspects of health were addressed in the ICECAP-O. In sum, this signifies improved understanding of the capability perspective in general for older persons and attained content validity for the ICECAP-O measure. Hence, the Swedish translation of the ICECAPO appears to hold content validity (Fitzpatrick et al., 1998). Items indeed measure the supposed domains; QoL with a capability approach. The questionnaire can therefore be further tested in practice in Sweden.

Finally, an improved questionnaire format with larger text size to meet the needs of visually impaired persons was advocated. This is a simple adjustment that would increase usability and the convenience for older persons, and is therefore recommended. Also, in line with a previous argument and a proposal by Horwood et al. (Horwood et al., 2014), we suggest the addition of a short statement in parentheses after each attribute with a description or example clarifying the meaning of each attribute. This would be a possible solution clarifying the concerns regarding Control.

Issues regarding this study's methodology should be raised. First, 18 older persons were recruited, which is concordant with Lee's (Lee, 2014) recommended sample size of approximately 15 participants in cognitive interviews within cross-cultural research. However, in addition to being 70 years old and community dwelling, participants were in relatively good health, lived in the same city, had a generally high education level and only two lived alone. We can also assume that older persons born outside Sweden with a lower Swedish proficiency are underrepresented because the study's invitation letter was written in Swedish. These factors combined with the fact that the result show that participants had rather high index scores on ICECAP-O, imply that our study entailed a relatively homogenous group of participants, which may have impacted the results. For instance, persons with lesser ability to speak Swedish or complex health needs might struggle more while competing the ICECAP-O. We thus recommend further evaluation of face and content validity and also acceptability by including more diverse groups of older persons in terms of age, health status, and ability to speak Swedish, as well as including persons living in senior housing and rural areas.

Second, the translation of key concepts in a questionnaire is an important topic. In our study, we used an internationally recognized procedure of translation (Guillemin et al., 1993), and involved a panel of experts from the field in order to obtain the final version of the translated ICECAP-O measure. Even so, minor discrepancies in the translation may have occurred. A potential 
example is the word pleasure in the operationalization of the fourth attribute: Enjoyment - enjoyment and pleasure. It seems like the translated Swedish word for pleasure can be associated with sex. This resulted in some participants raising this subject when discussing Enjoyment rather than Attachment - love and friendship, which more often raised discussions of intimacy and sex (Horwood et al., 2014). Nevertheless, this occurrence can also be explained by a participant's individual preferences and should thus not induce immediate modifications, but should rather be considered in future evaluation.

Finally, we highlight the approach used to analyze the cognitive interviews. We chose, in line with others (García, 2011; Horwood et al., 2014), to use a mixed approach; both a standardized classification scheme to quantify response problems and a qualitative thematic analysis to capture participants' experiences. This mixed approach is advantageous because it contains both subjective and objective aspects (Onwuegbuzie, Johnson, \& Collins, 2011) and counters criticisms levelled against cognitive interviews of being overly subjective (Drennan, 2003). To further address the matter of subjectivity, we employed three raters who independently coded data and we only registered a judged problem when two or more of the raters identified an equal problem. In addition, a limitation might be that our thematic analysis was performed on a manifest level; deeper underlying meanings on a latent level have not been explored. Furthermore, the use of PA for analyzing interrater agreement can be questioned, and Kappa values may be proposed as an enhanced option. However, we refer to researchers stating that PA is a sufficient method in preconditions similar to those in our study (McHugh, 2012; Svensson, 2001).

In sum, cognitive interviews with a mixed approach to data analyses proved to be a valuable method for evaluating face and content validity and acceptability for the ICECAP-O capability measure. However, considering possible methodological shortcomings, we recommend further research engaging a more varied group of older persons.

\section{Conclusions}

The ICECAP-O appears to measure what it is supposed to measure, QoL with a capability approach, and acceptability is satisfactory. Even so, the attribute Control - (i.e., independence) might be considered in need of a minor overhaul for use in the Swedish context, and the layout adapted for visually impaired persons is desirable. The ICECAP-O has potential for becoming a valuable addition to the supply of QoL measures in research and health and social care in Sweden. However, we recommend further research on a more diverse group of older persons.

\section{References}

Baltes, P. B., \& Smith, J. (2003). New frontiers in the future of aging: From successful aging of the young old to the dilemmas of the fourth age. Gerontology, 49(2), 123-135.

Braun, V., \& Clarke, V. (2006). Using thematic analysis in psychology. Qualitative Research in Psychology, 3(2), 77-101. doi:10.1191/1478088706qp063oa

Coast, J., Flynn, T. N., Natarajan, L., Sproston, K., Lewis, J., Louviere, J. J., \& Peters, T. J. (2008). Valuing the ICECAP capability index for older people. Social Science and Medicine, 67(5), 874-882. doi:10.1016/j.socscimed.2008.05.015
Coast, J., Peters, T. J., Natarajan, L., Sproston, K., \& Flynn, T. (2008). An assessment of the construct validity of the descriptive system for the ICECAP capability measure for older people. Quality of Life Research, 17(7), 967-976. doi:10.1007/ s11136-008-9372-z

Couzner, L., Ratcliffe, J., Lester, L., Flynn, T., \& Crotty, M. (2013). Measuring and valuing quality of life for public health research: Application of the ICECAP-O capability index in the Australian general population. International Journal of Public Health, 58(3), 367-376. doi:10.1007/s00038-012-0407-4

Drennan, J. (2003). Cognitive interviewing: Verbal data in the design and pretesting of questionnaires. Journal of Advanced Nursing, 42(1), 57-63. doi:10.1046/j.1365-2648.2003.02579.x

Ekelund, C., Dahlin-Ivanoff, S., \& Eklund, K. (2014). Self-determination and older people-A concept analysis. Scandinavian Journal of Occupational Therapy, 21(2), 116-124. doi:10.3109/11038128.2013.853832

Fitzpatrick, R., Davey, C., Buxton, M. J., \& Jones, D. R. (1998). Evaluating patient-based outcome measures for use in clinical trials. Health Technology Assessment, 2(14), i-74.

García, A. A. (2011). Cognitive Interviews to Test and Refine Questionnaires. Public Health Nursing, 28(5), 444-450. doi:10.1111/j.1525-1446.2010.00938.x

Garratt, A., Schmidt, L., Mackintosh, A., \& Fitzpatrick, R. (2002). Quality of life measurement: Bibliographic study of patient assessed health outcome measures. British Medical Journal, 324(7351), 1417-1419.

Grewal, I., Lewis, J., Flynn, T., Brown, J., Bond, J., \& Coast, J. (2006). Developing attributes for a generic quality of life measure for older people: Preferences or capabilities? Social Science and Medicine, 62(8), 1891-1901. doi:10.1016/j.socscimed.2005.08.023

Guillemin, F., Bombardier, C., \& Beaton, D. (1993). Cross-cultural adaptation of health-related quality of life measures: Literature review and proposed guidelines. Journal of Clinical Epidemiology, 46(12), 1417-1432. doi:10.1016/08954356(93)90142-N

Hammell, K. W. (2015). Quality of life, participation and occupational rights: A capabilities perspective. Australian Occupational Therapy Journal, 62(2), 78-85. doi:10.1111/ 1440-1630.12183

Horwood, J., Sutton, E., \& Coast, J. (2014). Evaluating the Face Validity of the ICECAP-O Capabilities Measure: A "Think Aloud" Study with Hip and Knee Arthroplasty Patients. Applied Research in Quality of Life, 9(3), 667-682. doi:10.1007/s11482-013-9264-4

Hörder, H., Gustafsson, S., Rydberg, T., Skoog, I., \& Waern, M. (2016). A Cross-Cultural Adaptation of the ICECAP-O: TestRetest Reliability and Item Relevance in Swedish 70-YearOlds. Societies, 6(4), 30.

Lee, J. (2014). Conducting Cognitive Interviews in Cross-National Settings. Assessment, 21(2), 227-240. doi:10.1177/ 1073191112436671

Makai, P., Brouwer, W. B. F., Koopmanschap, M. A., Stolk, E. A., \& Nieboer, A. P. (2014). Quality of life instruments for economic evaluations in health and social care for older people: A systematic review. Social Science and Medicine, 102, 83-93. doi:10.1016/j.socscimed.2013.11.050

McHugh, M. L. (2012). Interrater reliability: the kappa statistic. Biochemia Media (Zagreb), 22(3), 276-282.

Onwuegbuzie, A. J., Johnson, R. B., \& Collins, K. M. T. (2011). Assessing legitimation in mixed research: A new framework. Quality and Quantity, 45(6), 1253-1271. 
Prah Ruger, J. (2010). Health Capability: Conceptualization and Operationalization. American Journal of Public Health, 100(1), 41-49. doi:10.2105/AJPH.2008.143651

Rinder, L., Roupe, S., Steen, B., \& Svanborg, A. (1975). Seventy year old people in Gothenburg. A population study in an industrialised Swedish city. I. General presentation of the study. Acta Medica Scandinavica, 198(5), 397-407.

Sen, A. (1993). Capability and well-being (A. Sen Ed.). Oxford: Clarendon.

Sen, A. (2009). The idea of justice. Belknap Press of Harvard University Press.

Svensson, E. (2001). Guidelines to statistical evaluation of data from rating scales and questionnaires. J Rehabil Med, 33(1), 47-48.

Van Leeuwen, K. M., Jansen, A. P. D., Muntinga, M. E., Bosmans, J. E., Westerman, M. J., Van Tulder, M. W., \& Van Der Horst, H. E. (2015). Exploration of the content validity and feasibility of the EQ-5D-3L, ICECAP-O and ASCOT in older adults. BMC Health Services Research, 15(1). doi:10.1186/s12913015-0862-8

Willis, G. B., \& Miller, K. (2011). Cross-cultural cognitive interviewing: Seeking comparability and enhancing understanding. Field Methods, 23(4), 331-341. doi:10.1177 /1525822X11416092. 